\title{
Plantæ Sinenses Yoshianæ.
}

\section{III.}

Auctore

\section{Tokutaro Ito.}

\section{PORTULACACE $\not$.}

Portulaca oleracea, Linn. Sp. Pl. ed. 1, p. 445 ; Thunb. Fl. Jap. p. 192 ; DC. Prodr. III, p. 353 ; Lour. Fl. Cochinch. ed. Willd. p. 359 ; Ledeb. Fl. Ross. II, p. 145 ; Bunge, Enum. Pl. Chin. Bor. p. 104 ; Seemann, Bot. Voy. 'Herald,' p. 379 ; Benth. Fl. Hongk. p. 127; Maxim. Primit. Fl. Amur. pp. 113 et 472 ; Regel, Tent. Fl. Ussur. p. 64; Franch. et Sav. Enum. Pl. Jap. I, p. 53 ; Dyer in Hook. f. Fl. Brit. Ind. I, p. 246 ; Nyman, Consp. p. 253 ; Debeaux, Fl. Tchef. p. 63 ; Forbes et Hemsl. in Journ. Linn. Soc. XXIII, p. 71 ; Henry in Trans. As. Soc. Jap. XXIV, suppl. p. 19 ; Gürke, Pl. Europ. II, p. 1'79; T. Ito et Matsumura, T'ent. F]. Lutch. p. 50.

馬[蔽]莞 Bakin [recte Bashiken], vulgo Uma Biju, it. Siberi Fiju [recte Suberi Biyu], Kiempf. Amœn. p. 831.

Hab. in provincia Shantung: ad urbem Tshifu, mense Octobris 1897, fructif. (Domina Yoshi T'akagaki, n. 89!).

Observ. Portulaca grandiflora, Hook., planta austro-americana, colitur in hortis urbis Tshifu (Domina Yoshi Takagaki, n. 88 !).

\section{CARYOPHYLLACE $A$.}

Arenaria serpyllifolia, Linn. Sp. Pl. ed. 1, 423; DC. Prodr. I, p. 411 ; Fenzl in Ledeb. Fl. Ross. I, p. 368 ; Maxim. in Mél. Biol. IX, p. 34, Enum. Pl. Mongol. p. 98, et in Act. Hort. Petrop. XI, p. 69 ; Franch. Pl. David. I. p. 53, II, p. 205 ; Forbes et Hemsl. in Journ. Linn. Soc. I, p. 70 ; F. N. Williams, in Journ. Linn. Soc. XXXIII, p. 365, XXXIV, p. 436 ; Nyman, Consp. p. 115 ; Gürke, Pl. Europ. II, p. 272.

Hab. in provincia Shantung: in colle Tshifu-shan, 3 Aprilis 1898 (Domina Yoshi Takagaki, n. 557 !). 
Dianthus sinensis, Linn. Sp. Pl. ed. 1, p. 411 ; DC. Prodr. I, p. 359 (sensu latiore); Forbes et Hemsl. in Journ. Linn. Soc. XXIII, p. (63) (chinensis); Maxim. Ind. Fl. Mongol. in Primit. Fl. Amur. p. 480, Enum. Pl. Mongol. p. 84 (chinensis), et in Act. Hort. Petrop. XI, p. 64; F. N. Williams, in Journ. Linn. Soc. XXIX, p. 429 ; T. Ito et Matsumura, Tent. Fl. Lutch. p. 45 .

D. Seguieri, Chaix apud Vill.; Ledeb. Fl. Ross. I, p. 277 ; Koch, Syn. Fl. Germ. Helv. p. 104; Regel, Tent. Fl. Ussur. p. 25, et in Bull. Soc. Nat. Mosc. 1861, 4, p. 524 ; Edgew. et Hook. f. in Hook. f. Fl. Brit. Ind. I, p. 215 ; Maxim. in Bull. Soc. Nat. Mosc. 1879, p. 6 ; Nyman, Consp. p. 101 ; Franch. Pl. David. I, p. 45.

var. sylvaticus, Rohrb. subsp. brachylepis, Rohrb.; Maxim. Enum. Pl. Mongol. p. 84 ; F. N. Williams, in Journ. Linn. Soc. XXIX, p. 430.

D. Seguieri var. sylvestris, Koch, Syn. Fl. Germ. Helv. p. 104; Regel, Tent. Fl. Ussur. p. 26, et in Bull. Soc. Nat. Mosc. 1861, 4, p. 524.

Hab. in provincia Shıntung: Yantai prope urbem T'shifu, mensibus Octobris et Novembris 1897, nn. 60 et 117, flor. cum fruct. mat., in colle T'shifu-shan, 25 Septembris 1897 , n. 235, flor. cum fruct. mat., in collibus Nansi-shan, 17 Octobris 1897, n. 262, et Nanshan, 31 Octobris 1897, n. 343, flor. et fructif., nec non sub monte Si-shan (Temple Hills), 24 Octobris 1897, n. 426, flor. cum fruct. mat. (Domina Yoshi Takagaki!).

Perennis cespitosus glabrescens. Caules erecti vel ascendentes, $10-24 \mathrm{~cm}$. alti, elongati tetragoni apice dichotome ramosi vel uniflori. Folia linealilanceolata vel linearia, $10-50 \mathrm{~mm}$. longa, 1-5 mm. lata, plana rigida glabrescentia acuminata rarius acutiuscula basi contorta stricta 3- vel 5-nervia margini hispida, vagina folii 1-3 mm. longa. Flores $15-20 \mathrm{~mm}$. diam. solitarii vel gemminati vel in paniculis laxe dispositi. Bractere 4-6, incequales atting. $\frac{1}{2}-\frac{1}{3}$ calycis longitudinem, interiores oblongæe vel obovatie longe acuminatæ, externe ovales subulato-acuminate. Calyx tubo cylindrico multistriato $15-20 \mathrm{~mm}$. longo, dentibus deltoideo-lanceolatis acutis vel subacuminatis margini membranaceis pubescentibus. Petala rosea obovato-spatulata 20-30 mm. longa, antice irregulariter dentata, basi anguste attenuata. Anthere purpureascentes. Capsula cylindrica breve petiolata vel subsessilis, glaberrima, 15-20 $\mathrm{mm}$. longa. Semina nigrescentia minute granulata, $2 \mathrm{~mm}$. diam.

Gypsophila Oldhamiana, Miq. in Ann. Mus. Bot. Lugd. Bat. III, p. 187, Prol. Fl. Jap. p. 351 ; Maxim. in Mél. Biol. IX, p. 55, in adnot.; Forbes et Hemsl. in Journ. Linn. Soc. XIII, p. 64; F. N. Williams, in Journ. Bot. 1889, p. 325. 
G. altissima, Old. sec. F. N. Williams, loc. cit. (non L.).

Hal. in provincia Shantung: ad urbem Tshifu, mense Aprilis 1898, steril., n. 546, ad Yantai, mense Octobris 1897, steril., n. 443, nec non ad Nansi-shan, 17 et 31 Octobris 1897, flor. et fructif., nn. 265, 356 (Domina Yoshi Takagaki!).

Glaberrima glauca. Candex suffruticosus sepe crassus ramosus. Caule erecti fere $30-50 \mathrm{~cm}$. alti cylindrici vel subangulati tenues firmis nodosis, inferne simplices, superne paniculato-ramosi, ramis floridis. Folia caulina oblonga vel oblongo-lanceolata, 25-65 mm. longa, 5-12 mm. lata, acuta vel apiculata basi attenuata 3-5-nervia; ramulorum floridorum diminuta linearia vel subulata. Cyme multiflore corymbose 2-4-cm. late. Flores fere $5 \mathrm{~mm}$. diam. pedicellati, pedicello filiformi $2-5 \mathrm{~mm}$. longo calyce sequilongo vel longiori. Bracteole ovatee cuspidate vel acuminatie scariose 1-2mm. longe. Calyx $2 \frac{1}{2}-3 \mathrm{~mm}$. longus, $1 \frac{1}{2}-2 \mathrm{~mm}$. latus, turbinatus minute glandulosopunctatus quinquenervius preter nervos scariosus, dentibus obtiusculis. Petali alba, 4-5 mm. longa, $1 \mathrm{~mm}$. lata, cuneato-oblonga truncata obtusa trinervia. Stamina filiformia $5 \mathrm{~mm}$. longa, antheri globosa. Styli 2 , filiformes, $4 \mathrm{~mm}$. longi. Ovarium obovoideum glabrum vix $1 \mathrm{~mm}$. longum; ovula fere 12. Capsula glabra globosa, $2 \frac{1}{2} \mathrm{~mm}$. diamet. Semina subreniformia crebre tuberculata rufo-brunnea, $1 \frac{1}{3} \mathrm{~mm}$. longa, $1 \frac{1}{4} \mathrm{~mm}$. lata.

\section{Catalogus plantarum ad stationem zoologicam Misakensem sponte crescentium.}

Scripsit

Y. Yabe.

II.

\section{DICOTYLEDONEAE.}

Saururaceæ.

Houttuynia corda, Thunb. ドクダミ

\section{Piperaceæ.}

Piper Futo-kadsura, $S$. et $Z$. フゥトロカジラ 